\title{
A NOVEL QUERY DRIVEN POWER-BALANCED ROUTING PROTOCOL FOR WSN
}

\author{
Ayan Kumar Das ${ }^{1}$ and Dr. Rituparna Chaki ${ }^{2}$ \\ ${ }^{1}$ Department of Information Technology, Calcutta Institute of Engineering and \\ Management, Kolkata, India, \\ ayandas24114057@yahoo.co.in \\ ${ }^{2}$ Department of Computer Science \& Engineering, West Bengal University of \\ Technology, Kolkata, India, \\ rituchaki@gmail.com
}

\begin{abstract}
Wireless sensor networks consist of hundreds or thousands of small sensors with have limited resources. The energy resource is the most challenging one to be maintained. The major reason for power drain is the communication between sensor nodes. Most of the routing algorithms for sensor networks focus on finding energy efficient paths to prolong the lifetime of the networks. In this paper, a novel routing algorithm has been proposed to detect the source of an event in the network. This algorithm also maintains a balance between the powers of different nodes in the network, so that the longevity of the overall network may increase.
\end{abstract}

\section{KEYWORDS}

update sensing, event path, query node, Infinite loop.

\section{INTRODUCTION}

Sensor networks are among the fastest growing technologies that have the potential of changing our lives drastically. These collaborative, dynamic and distributed computing and communicating systems will be self organizing. They will have capabilities of distributing a task among themselves for efficient computation. A Wireless Sensor Network (WSN) contains hundreds or thousands of sensor nodes. These sensors have the ability to communicate either among each other or directly to an external base-station (BS). A greater number of sensors allows for sensing over larger geographical regions with greater accuracy.

Despite the innumerable applications of WSNs, these networks have several restrictions, e.g., limited energy supply, limited computing power, and limited bandwidth of the wireless links connecting sensor nodes. One of the main design goals of WSNs is to carry out data communication while trying to prolong the lifetime of the network and prevent connectivity degradation by employing aggressive energy management techniques. The design of routing protocols in WSNs is influenced by the above challenging factors. These factors must be overcome before efficient communication can be achieved in WSNs. The computation involving sensed data and communication of the same between the sensor nodes cause very high power consumption. This coupled with the inability to recharge once a node is deployed makes energy consumption which is the most important factor to determine the life of a sensor network, because usually sensor nodes are driven by battery and have very low energy resources. This can be achieved by having energy awareness in every aspect of design and operation of the network. The common routing techniques are based on power awareness, agent based, location based etc. To prolong the lifetime of sensor networks, 
most routing algorithm for sensor networks focus on finding energy efficient paths. As a result, the sensors on the efficient paths are depleted very early, and consequently the sensor networks become incapable of monitoring events from some of the parts of target areas.

It has been observed that most of the previous routing techniques do not maintain any information about the nodes which have been already traversed. Thus a query packet moving towards the event path randomly selects neighboring nodes without considering whether that node has already been traversed. This often leads to the infinite loop problem, leading to increased delay in routing. This paper proposes a query driven routing protocol with optimal power balancing techniques aimed at removing the infinite loop problem.

The remaining part of this paper is organized as follows: Section 2 deals with the review of state of the art routing topologies, section 3 gives a description of the proposed methodology, section 4 contains the simulation reports and section 5 is the concluding part.

\section{REVIEW WORKS OF SENSOR NETWORKS}

In this section, we survey the state-of-the-art routing protocols for WSNs. In general, routing in WSNs is categorized into power aware routing, query driven routing, zone based routing, agent based routing and cluster based routing.

\subsection{Power Aware Routing Algorithm}

This type of routing technique focuses on the effect of power efficient routing on the lifetime of multi hop wireless sensor networks (WSNs) [5]. All the nodes in the WSNs are divided into tiers. The nodes belonging to the highest tier are very important, because these nodes imply higher power consumption than that of nodes of any other tier. Obviously the batteries of nodes of $1^{\text {st }}$ tier depletes sooner than these of any other tier. As soon as the nodes of $1^{\text {st }}$ tier dies, the network becomes disconnected.

Reliable Energy Aware Routing (REAR) [8] is a distributed, on-demand, reactive routing protocol and is used to provide a reliable transmission environment for data packet delivery. To provide a reliable transmission environment to reduce retransmissions caused by unstable paths REAR introduces local node selection, path reservation and path request broadcasting delay. This algorithm efficiently utilizes the limited energy and available memory resources of sensor nodes. REAR takes precaution against errors, instead of finding a solution for the errors. The Simulation experiments show that REAR outperforms traditional schemes by establishing an energy-sufficient path from the sink to the source with special path request flooding, and also by distributing the traffic load more evenly in the network.

An approximation algorithm called max-min zPmin [27] has developed with a good empirical competitive ratio. This algorithm combines the benefits of selecting the path with the minimum power consumption and the path that maximizes the minimal residual power in the nodes of the network.

Minimum Cost Forwarding Algorithm (MCFA) [14] exploits the fact that the direction of routing is always known, that is, towards the fixed external base-station. Hence, a sensor node need not have a unique ID nor maintain a routing table. Instead, each node maintains the least cost estimate from itself to the base-station. Each message to be forwarded by the sensor node is broadcast to its neighbors. When a node receives the message, it checks if it is on the least cost path between the source sensor node and the base-station. If this is the case, it rebroadcasts the message to its neighbors. This process repeats until the base-station is reached. 
GRAdient Broadcast [24] describes a technique of building a cost field toward a particular sensor node, and after that reliably routing query across a limited size mesh toward that sensor node. Overhead comes for a network flood to set up the cost field, but queries can be routed along an interleaved set of short paths, and can thus be delivered very cheaply and reliably. GRAB was not designed specifically to support in network processing but significantly influenced the work presented in its use of event-centric routing state in the network.

Directed Diffusion and Geo-Routing [19][21][22] provides a scheme for doing a limited flood for a query toward the event, and then setting up reverse gradients in order to send data back along the best route. Though Diffusion results in high quality paths, but an initial flood of the query for exploration will be the requirement. One of its primary contributions is an architecture that names data and that is intended to support in network processing. Rumor routing is intended to work in conjunction with diffusion, bringing innovation from GRAB and GOSSIP routing to this context.

Constrained Anisotropic Diffusion Routing (CADR) [25] aims to be a general form of directed diffusion. The key idea is to query sensors and route data in the network such that the information gain is maximized while latency and bandwidth are minimized. CADR diffuses queries by using a set of information criteria to select which sensors can get the data. This is achieved by activating only the sensors that are close to a particular event and dynamically adjusting data routes. The main difference from directed diffusion is the consideration of information gain in addition to the communication cost. In CADR, each node evaluates an information/cost objective and routes data based on the local information/cost gradient and enduser requirements. Estimation theory was used to model information utility measure.

COUGAR [26] is a data-centric protocol which views the network as a huge distributed database system. The key idea is to use declarative queries in order to abstract query processing from the network layer functions such as selection of relevant sensors and so on. COUGAR utilizes in-network data aggregation to obtain more energy savings. The abstraction is supported through an additional query layer that lies between the network and application layers. COUGAR incorporates architecture for the sensor database system where sensor nodes select a leader node to perform aggregation and transmit the data to the BS. The BS is responsible for generating a query plan, which specifies the necessary information about the data flow and in network computation for the incoming query and send it to the relevant nodes. The query plan also describes how to select a leader for the query. The architecture provides in-network computation ability that can provide energy efficiency in situations when the generated data is huge. COUGAR provided network-layer independent methods for data query. However, COUGAR has some drawbacks. First, the addition of query layer on each sensor node may add an extra overhead in terms of energy consumption and memory storage. Second, to obtain successful in-network data computation, synchronization among nodes is required (not all data are received at the same time from incoming sources) before sending the data to the leader node. Third, the leader nodes should be dynamically maintained to prevent them from being hot-spots.

\subsection{Query Driven Routing}

In Information-Driven Sensor Querying (IDSQ) [25], the querying node can determine which node can provide the most useful information with the additional advantage of balancing the energy cost. However, IDSQ does not specifically define how the query and the information are routed between sensors and the BS. Therefore, IDSQ can be seen as a complementary optimization procedure. Simulation results showed that these approaches are more energyefficient than directed diffusion where queries are diffused in an isotropic fashion and reaching nearest neighbors first. 
Rumor routing [18] is a variation of directed diffusion and is mainly intended for applications where geographic routing is not feasible. In general, directed diffusion uses flooding to inject the query to the entire network when there is no geographic criterion to diffuse tasks. However, in some cases there is only a little amount of data requested from the nodes and thus the use of flooding is unnecessary. An alternative approach is to flood the events if the number of events is small and the number of queries is large. The key idea is to route the queries to the nodes that have observed a particular event rather than flooding the entire network to retrieve information about the occurring events. In order to flood events through the network, the rumor routing algorithm employs long-lived packets, called agents. When a node detects an event, it adds such event to its local table, called events table, and generates an agent. Agents travel the network in order to propagate information about local events to distant nodes. When a node generates a query for an event, the nodes that know the route, may respond to the query by inspecting its event table. Hence, there is no need to flood the whole network, which reduces the communication cost. On the other hand, rumor routing maintains only one path between source and destination as opposed to directed diffusion where data can be routed through multiple paths at low rates. Simulation results showed that rumor routing can achieve significant energy savings when compared to event flooding and can also handle node's failure. However, rumor routing performs well only when the number of events is small. For a large number of events, the cost of maintaining agents and event-tables in each node becomes infeasible if there is not enough interest in these events from the BS. Moreover, the overhead associated with rumor routing is controlled by different parameters used in the algorithm such as time-to-live (TTL) pertaining to queries and agents. Since the nodes become aware of events through the event agents, the heuristic for defining the route of an event agent highly affects the performance of next hop selection in rumor routing.

\subsection{Zone Based Routing}

The zone-based routing [27] algorithm relies on max-min zPmin. It is scalable for large scale networks and is used to optimize the lifetime of the network. Zone-base routing is a hierarchical approach where the area covered by the sensor network is divided into a small number of zones. Each zone has many nodes and thus a lot of redundancy occurs in routing a message through it. To send a message across the entire area it finds a global path from zone to zone and gives each zone control over how to route the message within itself. A local path for the message is computes within each zone so as to not decrease the power level of the zone too much

\subsection{Agent based Routing Algorithm}

Apart from power aware routing some algorithms modeled after agent behavior. Agents traverse the network encoding the quality of the path they are traveled, and leave it the encoded path as state in the nodes [23]. In dealing with failure these algorithms are very effective, as there is always some amount of exploration, and especially around previously good solutions. However, due to the large number of nodes, the number of ant agents required to achieve good results tends to be very large, making them difficult to apply in sensor networks.

Ant-Colony Based Routing Algorithm (ARA) [17] used distance vector routing. Route discovery in ARA is done by broadcasting Forward Ants (FANT). ARA implements the packet sequencing mechanism to prevent packet loops. In ARA, destinations respond to multiple FANT packets received from the same node, thus supporting multi path routing.

MONSOON [1] [2] [3] proposed an evolutionary multi objective adaptation framework, in biologically inspired application architecture, called BiSNET/e. There are two types of software components- agents and middleware platforms. In MONSOON each application is implemented as a decentralized group of software agents, like a bee colony consists of bees. 
Agents generally collect all the sensing data and/or detect an event on individual nodes, and also carry sensing data to the base station. Here each agent decides their behavior based on logical energy. It does not represent the amount of physical battery in a node, as it is a logical concept.

\subsection{Cluster Based Routing Algorithm}

In clustered network, nodes are clustered in any form of hierarchical structure. The advantage of cluster based approaches are improving routing efficiency, scalability, supporting QOS and saving power consumption in sensor nodes. Clustering transforms a physical network into a virtual network of interconnected clusters.

Low Energy Adaptive Clustering Hierarchy (LEACH) [20] is a cluster-based protocol, which includes distributed cluster formation. LEACH randomly selects a few sensor nodes as cluster heads $(\mathrm{CHs})$ and rotates this role to evenly distribute the energy load among the sensors in the network. In LEACH, the cluster head $(\mathrm{CH})$ nodes compress data arriving from nodes that belong to the respective cluster, and send an aggregated packet to the base station in order to reduce the amount of information that must be transmitted to the base station. LEACH uses a TDMA/CDMA MAC to reduce inter-cluster and intra-cluster collisions. However, data collection is centralized and is performed periodically. Therefore, this protocol is most appropriate when there is a need for constant monitoring by the sensor network. A user may not need all the data immediately. Hence, periodic data transmissions are unnecessary which may drain the limited energy of the sensor nodes. After a given interval of time, a randomized rotation of the role of the $\mathrm{CH}$ is conducted so that uniform energy dissipation in the sensor network is obtained. The authors showed that only $5 \%$ of the nodes need to act as cluster heads.

\section{PROPOSED WORK}

\subsection{Basic Methodology}

From the review of recent routing topologies, it is observed that many of the existing techniques fail to take care of rapid power loss by some nodes while other nodes remain unused, retaining the full power level. There is a possibility of cut-off of some parts of the network due to this unbalanced use of power. Also, many of the routing algorithms suffer from infinite loop problem, causing a packet to return to the point of origin after a while, instead of reaching the destination. This section describes a new routing protocol with aimed at reducing these problems. The network is modeled as a set of densely distributed wireless sensor nodes. Each node maintains a list of its neighbors, as well as an event table, with forwarding information of all the events in its knowledge. A node adds an event to its event table after encounter it. Then the node will propagate that event information to its neighbors and then to neighbors of neighbors and so on. This continues up to a certain distance, as it is unnecessary and also power wasting to inform the event to all the nodes of the network. The path which has the information about the event is known as event path. Any node from the network can generate a query and search for the event path. If it found that then it can reach to the event source node along with the event path.

However a problem may arise if we don't maintain any status checking option for the already traversed nodes. As a result the query while moving towards the event path randomly selects neighboring nodes without considering whether the next node has already been checked. This may lead to traversal or checking of the same node more than once. As a result lot of energy and time is wasted and may lead to the procedure getting stuck in an infinite loop. That is why the algorithm adds a checked/unchecked status to all the nodes to identify the previously visited node so that the infinite loop may be avoided.

A problem still exists as in all the cases the next hop node is chosen randomly without considering the available power of the node. Thus, the node with the least available power may be selected randomly and repeatedly as the node to be traversed next. This will result in the reduction of power of a node, which already has the smallest power while keeping the power of 
other nodes (with higher power than the node already selected as the next hop) constant. As a result of which, there exists a high chance of exhausting the total power of a particular node(s), keeping the power of other nodes almost unaffected. To overcome this disadvantage the algorithm maintains a data structure, containing the power content of all the nodes and depending on the power of these nodes the next hop is selected. The algorithm selects the node with the maximum power as the next hop. As a result of which, the power level within the network remain balanced.

\section{Definitions:}

Definition 1: Power required for transmission is directly proportional to the size of the packet that is send through the network and also the distance traveled by the packet, i.e., $\mathrm{P} \alpha \mathrm{s} * \mathrm{~d}$ Thus, we can write $\mathrm{P}=\theta^{*} \mathrm{~s} * \mathrm{~d}$

Where, $\mathrm{s}=$ size of the packet that is send through the network $\mathrm{d}=$ distance traveled by the packet, and $\boldsymbol{\theta}$ is a constant.

Definition 2: The total consumed ENERGY(E)during the transmission of a packet can be defined as the sum of power required to transmit and receive the packet, i.e.,

$\mathrm{E}=\sum$ (Power required to transmit the packet, Power required to receive the packet) .

$=\mathrm{P} 1+\mathrm{P} 2$

$=(\theta * \mathrm{~s} 1 * \mathrm{~d} 1)+(\theta * \mathrm{~s} 2 * \mathrm{~d} 2)$

Where s, s1 and s2 are the packet size whose value will be supplied by the user, d1 is the distance between the sending node and the current node and $\mathrm{d} 2$ is the distance between the current node and the receiving node.

\subsection{Data Dictionary}

Table 1. Variables list

\begin{tabular}{|l|l|}
\hline Variable name & Description \\
\hline $\mathrm{N}$ & Total Number of nodes \\
\hline $\mathrm{A}$ & An array consists of the connections between the nodes \\
\hline $\mathrm{C}\left[\mathrm{a}_{0}, \mathrm{a}_{1}, \mathrm{a}_{2}, . . \mathrm{a}_{\mathrm{n}}\right]$ & Event path nodes are stored in this array \\
\hline Power[node_id,energy] & An array consists of initial power of each node \\
\hline Visit[node_id] & Visited nodes are stored in this array \\
\hline $\mathrm{P}_{\mathrm{i}}[$ node_id, status] & Neighbors of node $\mathrm{i}$ are stored in this array along with their status. \\
\hline Next_node & next node chosen during the traversal \\
\hline Dist & the distance between the last and second last visited nodes. \\
\hline Tot & total distance traveled during one simulation \\
\hline Node_max_pow[ ] & The neighboring nodes with the maximum power are stored in this array \\
\hline
\end{tabular}

\subsection{Description}

Step 1. Begin.

Step 2. Read size (for the packet)

Step 3: Find the neighboring nodes of source node (m) and store them in the array 'p'.

Step 4. Repeat the following statements $n$ times (where $n$ is the number of neighbor nodes of source $m$ ) If status $\mathrm{i}=$ visited then delete $\mathrm{i}^{\text {th }}$ node from Visit[node_id]

Step 5. If all the neighboring nodes are visited previously, then Choose any one randomly.

Else Find the node among (not visited) nodes with the maximum power and store in the array 'node_max_power'.

Step 6. If more than one node exists with maximum power content, then select any of them randomly as the next hop and store it as 'next_node'.

Step 7. Use equation 2 to deduce remaining energy level 
Step 8. If the chosen next hop node falls on the event path then-calculate the distance traversed by the query to reach the event source and also calculate the average power reduction of each node for simultaneous queries.

Else Step 9. End.

Make the next hop node as the source node(m) and continue the process from step 3 .

\subsection{Case Study}

Consider the following wireless sensor network as shown in Figure 1 formed with 15 nodes. Let the node $\mathrm{n} 12$ generates the query and sends the query packet to node $\mathrm{n} 10$ or $\mathrm{n} 11$ which one has the maximum power. After that the packet will be moved to node $n 8$ and then from $n 8$ moves to $\mathrm{n} 9$ then to $\mathrm{n} 6$, from $\mathrm{n} 6$ to $\mathrm{n} 7$ and ultimately returns to the already traversed node $\mathrm{n} 8$ thereby creating an infinite loop and will never get the event path.

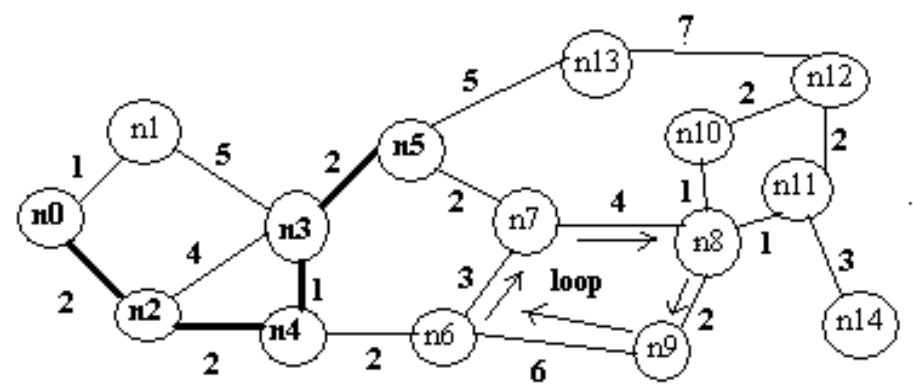

Figure 1. Infinite loop is formed

In order to overcome the above said limitation the proposed algorithm maintains a proper status checking to select the next hop node to avoid the infinite loop as shown in figure 2 .

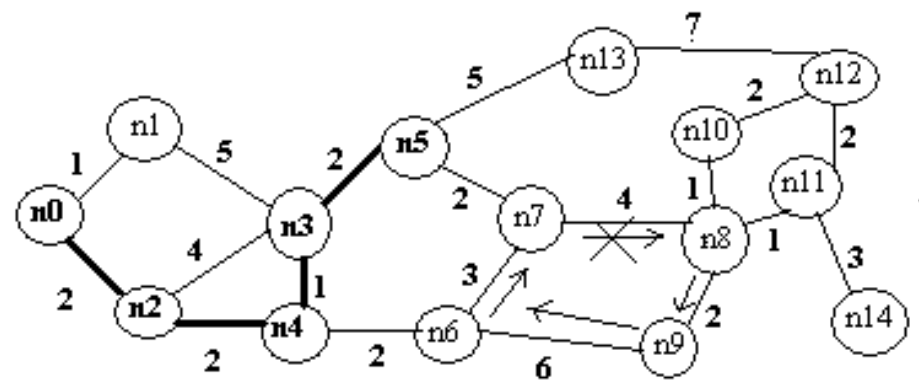

Figure 2. Infinite loop is not formed

Here since a track of all the traversed nodes were taken the packet which has started its journey from node $\mathrm{n} 8$ will not go back to same node $\mathrm{n} 8$ again after traversing node $\mathrm{n} 7$ as was the case in the previous example, instead it will move to node $\mathrm{n} 5$ as $\mathrm{n} 6$ and $\mathrm{n} 8$ are both already traversed nodes. Now node $\mathrm{n} 5$ is a node of event path and thus the packet will be able to reach at event source.

Through the network multiple simultaneous queries can be generated. For example if we send a query from node $\mathrm{n} 12$, then it will check the power of neighbor nodes $\mathrm{n} 10$ and $\mathrm{n} 11$ and select the node which contents of maximum power. If the selected node is $n 11$ then it will check the power of $n 8$ and $n 14$ and select the node of maximum power and so on. When it will encounter any node of event path then it will move to the event source directly along with the event path. 


\section{Simulation Result}

To analyze the performance of the algorithm multiple queries have been sent and after power consumption the power of all the nodes has measured and a graph of power vs node has drawn. It is also compared with the Rumor Routing algorithm. The parameter list is given below-

Table 2. Parameter list

\begin{tabular}{|l|l|}
\hline Parameters & Description \\
\hline Network size & 15 nodes \\
\hline Initial energy & 50J per node \\
\hline MAC Protocol & IEEE 802.15.4 \\
\hline $\begin{array}{l}\text { Power } \\
\text { consumption }\end{array}$ & $\begin{array}{l}\text { Equivalent to packet size } \\
\text { and distance }\end{array}$ \\
\hline $\begin{array}{l}\text { Number of } \\
\text { queries }\end{array}$ & At least 6 \\
\hline
\end{tabular}

According to the 'Rumor Routing Algorithm' where next hop node is choosing randomly, after sending 6 queries from node 15 the graph is shown below, where series 1 denotes the initial power of the node, series2-series6 denotes the power of the node after each query is generated, and series 7 denotes the average power of the nodes.

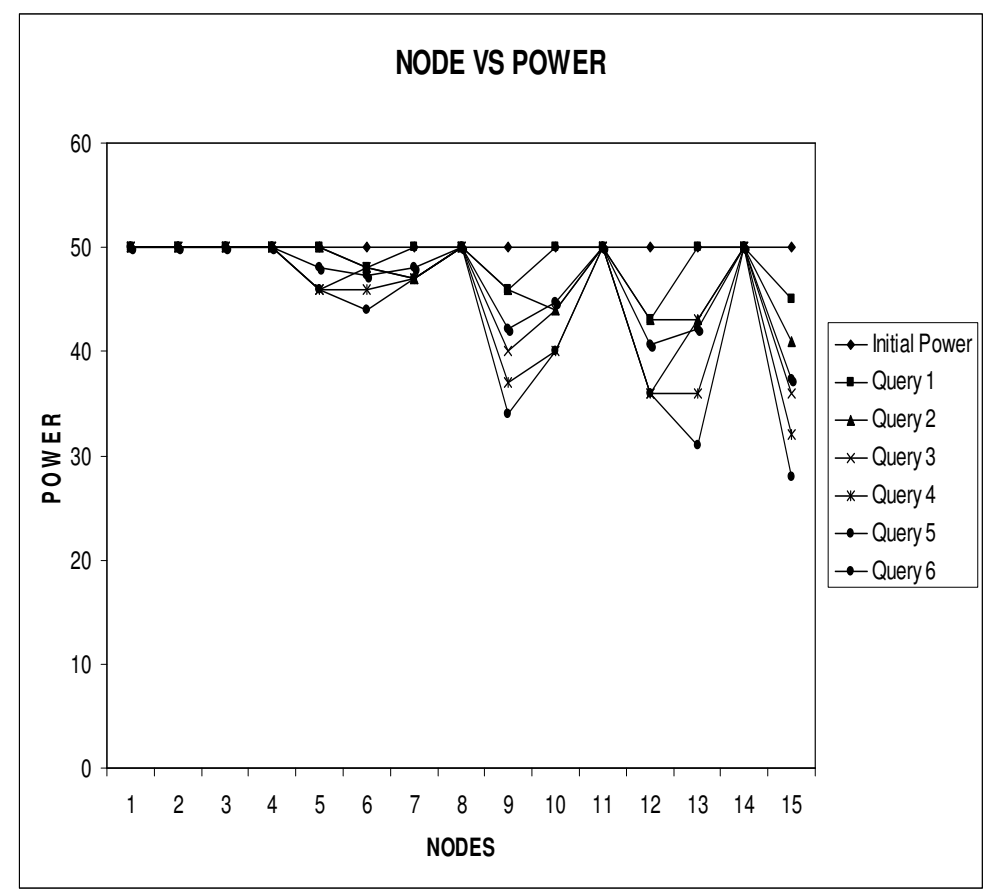

Figure 3. Node vs. Power graph for random neighbor selection

Now it is proposed that the next hop will be the node with the maximum power. When next hop is chosen based on the power of the neighboring nodes, the following graph is obtained, where series 1 denotes the initial power of the nodes, series2-series 6 denotes the power of the nodes after each query is generated, and series 7 denotes the average power of the nodes. 


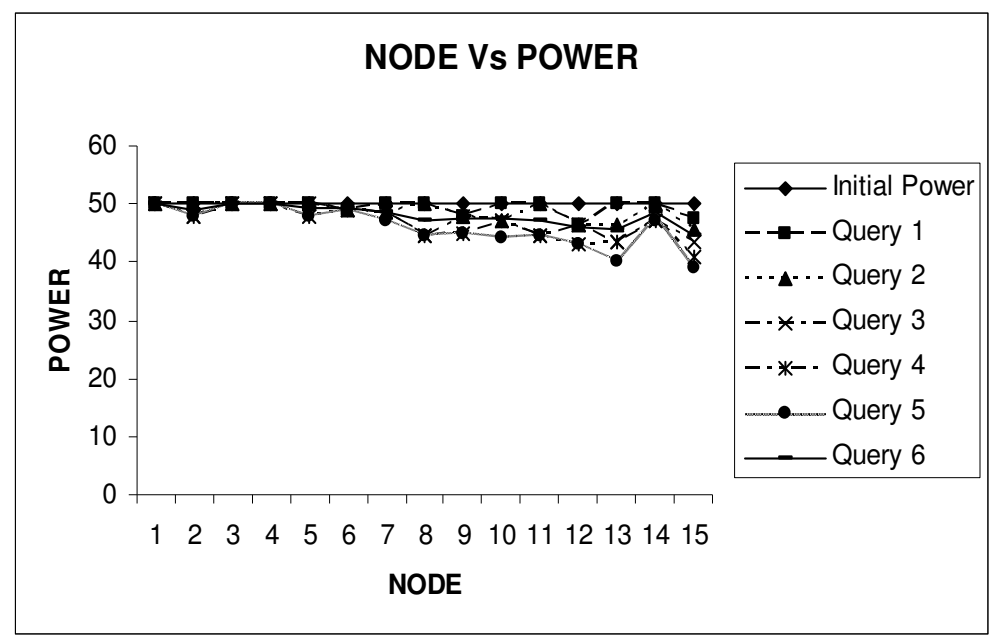

Figure 4. Node vs. Power graph for neighbor selection based on maximum power

Now, the average power of the nodes obtained in both are plotted in a graph, where series1 represents the average power of the node when the nodes are chosen randomly that is for Rumor Routing algorithm, series 2 represents the power of the nodes when the next hop is chosen based on the maximum power of the neighboring nodes.

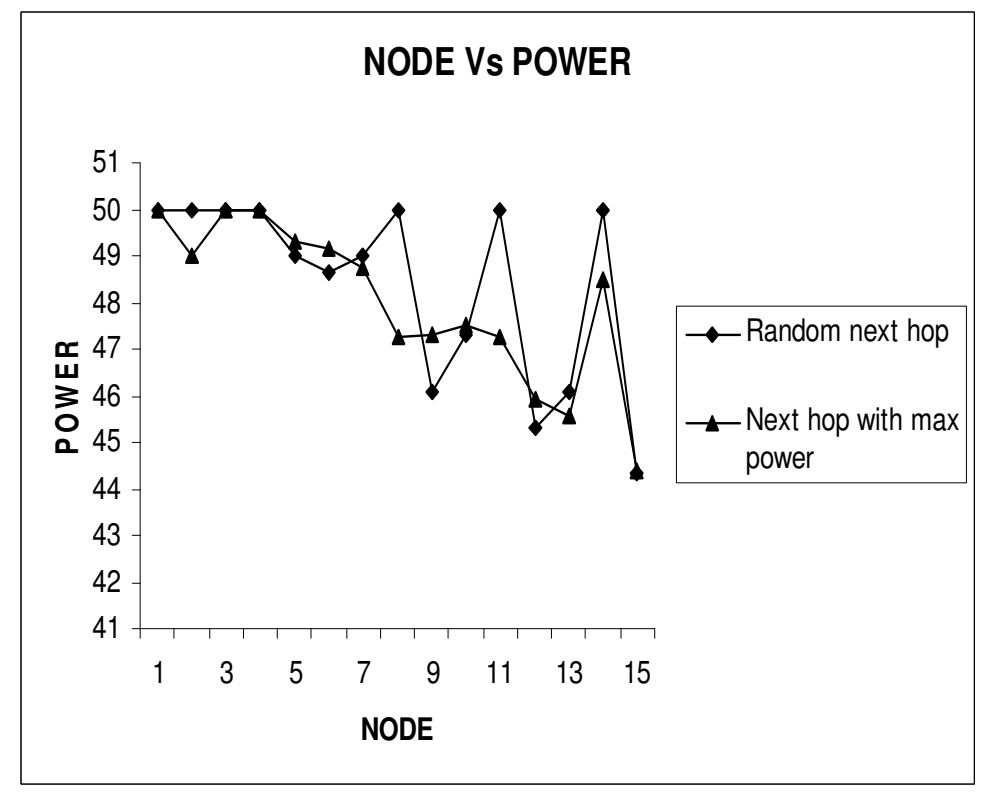

Figure 5. Node vs. Power graph by taking average power deduction for each node for random neighbor selection and neighbor selection based on maximum power 


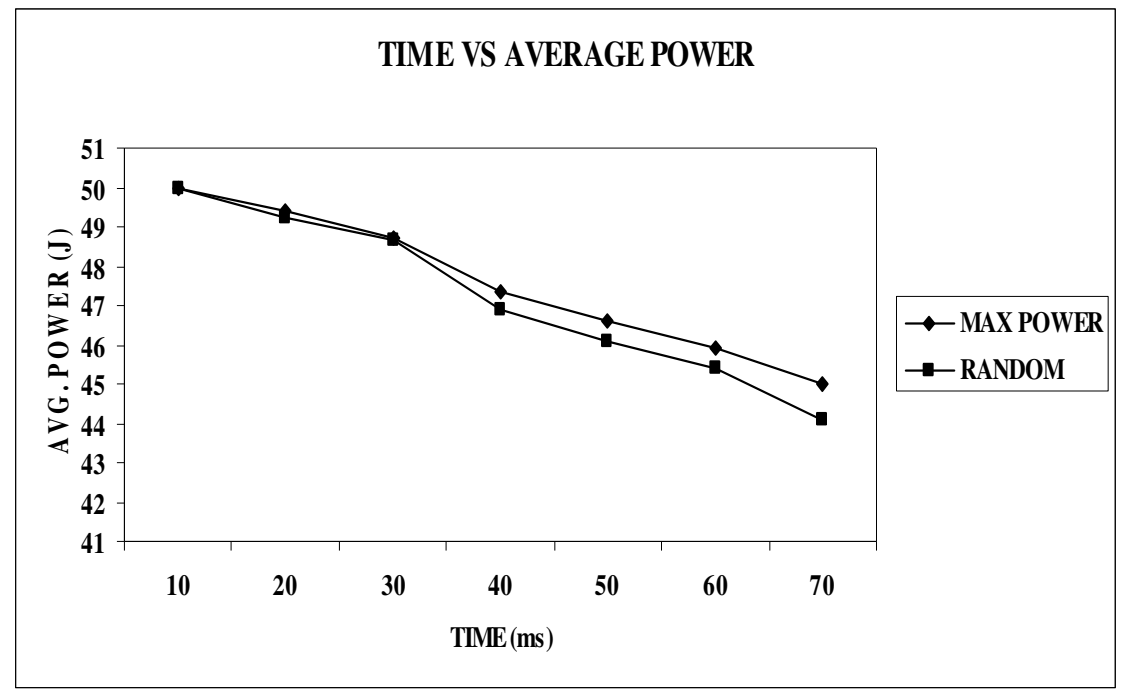

Figure 6. Average Power vs. Time graph for random neighbor selection and neighbor selection based on maximum power

In the above graph it is shown that when the time increases the average power deduction of the network for neighbor selection based on maximum power is less than that of random neighbor selection. Thus the network will be more stable if we choose the neighbor nodes based on maximum power contents and also the longevity of the network will be increased.

\section{CONCLUSION}

Sensor networks aim to achieve energy balance as well as energy efficiency. Till date, the energy-constrained nature of sensors poses the biggest problem in efficient management of sensor networks. The current state of the art is that for most of the power saving algorithms the cost factor is neglected, as they are not sending the packets in optimal path to balance the energy level between all the nodes in the network. This paper proposes an energy efficient technique to find out the source of the event, at the same time it selects the next hop node among the neighbor nodes which contain the maximum power. Thus there is a balance in power consumption for every node in the network. The simulation result shows the proposed algorithm increases the lifetime of every node in the network and thus it increases the longevity of the overall network.

\section{REFERENCES}

[1] PruetBoonma, and Junichi Suzuki, "MONSOON: A Co-evolutionary Multiobjective Adaptation Framework for Dynamic Wireless Sensor Networks", In Proceedings of the $41^{\text {st }}$ Hawaii International Conference on System Science, 2008.

[2] PruetBoonma, and Junichi Suzuki, "Exploring self-star Properties in Cognitive Sensor Networking", In Proc of IEEE/SCS International Symposium on Performance Evaluation of Computer and Telecommunication Systems (SPECTS), Edinburgh, UK, 2008.

[3] PruetBoonma, and Junichi Suzuki, "BiSNET: A biologically inspired middleware architechture for self managing wireless sensor networks", In Computer Networks, Vol.5.1, NO.16, pp. 4599-4616, 2007.

[4] Wei-Ming Chen, Chung-Sheng Li, Fu-Yu Chiang, Han-Chieh Chao "Jumping Ant Routing Algorithm for sensor networks", published by Elsvier B.V. in 2007. 
International Journal of Wireless \& Mobile Networks (IJWMN) Vol. 3, No. 4, August 2011

[5] Suyoung Yoon, RudraDutta and Mihail L. Sichitiu,"Power Aware Routing Algorithm for Wireless Sensor Networks" in IEEE 2007.

[6] Siva Kumar. D and Bhuvaneswaran.R.S, "Proposal on Multi agent Ants based Routing Algorithm for Mobile Adhoc Networks", in IJCSNS International Journal of Computer Science and Network Security, Vol-7, No. 6, June,2007.

[7] M.Dorigo, “Ant Colony Optimization”, University of Pretoria Etd, du Plessis J, 2006

[8] HossamHassaanein and Jing Luo "Reliable Energy Aware Routing in Wireless Sensor Networks" in IEEE in 2006.

[9] V.Laxmi, Lavina Jain, M.S.Gaur, "Ant Colony Optimization based Routing on NS-2" in the preceedings of International Conference On Wireless Communication and Sensor Networks, WCSN 2006

[10] Ace Abrenica, RezanAlmojulea, Roger Dalupang, RhodelleMagnayon (2005). "The Development of a ZigBee Wireless Home Area Network Utilizing Solar Energy as an Alternative Power Source". University of Manitoba.

[11] PaymanArabshahi, Andrew Gray, IoannisKassabalidis, Arindam Das, "Adaptive Routing in Wireless Communication Network using Swarm Intelligence", Jet Propulsion Laboratory and University of Washington.,2004

[12] KwangMongSim and Weng Hong Sun, Member IEEE, "Ant Colony Optimization or Routing and Load-Balancing: Survey and New Direction, part-A: System and Humans, Vol-33,No. 5 Sep,2003.

[13] Sundaram Rajagopalam, Chien-Chung Shen, "A Routing Suite for Mobile Ad hoc Networks using Swarm Intelligence”, Department of Computer and Information Sciences, University of Delaware, Newark,2003

[14] ] F. Ye, A. Chen, S. Liu, L. Zhang, \A scalable solution to minimum cost forwarding in large sensor networks", Proceedings of the tenth International Conference on Computer Communications and Networks (ICCCN), pp. 304-309, 2001.

[15] D. Gay, P. Levis, R. von Behren, M. Welsh, E. Brewer, and D. Culler. The nesC language: A holistic approach to networked embedded systems. In SIGPLAN Conference on Programming Language Design and Implementation (PLDI'03), June 2003.

[16] Dai, F., Wu. J. "Distributed dominant pruning in ad-hoc networks". In: Proceedings of ICC 2003.

[17] M. Gunes, U. Sorges, I. Bouazizi, “ARA- the ant colony based routing algorithm for MANET",ICPP Proc of the 2002

[18] David Braginsky and Deborah Estrin "Rumor Routing Algorithm for Sensor Networks" in WSNA '02, September 28, 2002.

[19] Yu, Y. Govindan, R. and Estrin, D. Geographical and Energy Aware Routing: A Recursive Data Dissemination Protocol for Wireless Sensor Networks. UCLA Computer Science Department Technical Report UCLA/CSD-TR-01-0023, 2001.

[20] W.R.Heinzelman, A.Chandrakasan, and H.Balakrishnan, "Energy Efficient Communication Protocol for Wireless Microsensor Networks", In Proceedings of the $33^{\text {rd }}$ Hawaii International Conference on System Sciences, 2000.

[21] Intanagonwiwat, C. Govindan R. and Estrin, D. Directed Diffusion: A Scalable and Robust Communication Paradigm for Sensor Networks. In Proceedings of the sixth Annual International Conference on Mobile Computing and Networks (MobiCOM2000), 2000 .

[22] Karp, B. and Kung, H.T. GPSR: Greedy perimeter stateless routing for wireless networks, In Proceedings of the ACM/IEEE International Conference on Mobile Computing and Networking, pages 243-254, boston, Mass., USA, 2000. 
International Journal of Wireless \& Mobile Networks (IJWMN) Vol. 3, No. 4, August 2011

[23] Subramanian, D. Druschel, P. Chen, J. Ants and Reinforcement Learning: A Case Study in Routing in Dynamic Data Networks. In Proceedings of IJCAI-1997.

[24] ]. C. Schurgers and M.B. Srivastava, "Energy efficient routing in wireless sensor networks", in the MILCOM Proceedings.

[25] ] M. Chu, H. Haussecker, and F. Zhao, "Scalable Information-Driven Sensor Querying and Routing for ad hoc Heterogeneous Sensor Networks," The International Journal of High Performance Computing Applications, Vol. 16, No. 3, August 2002.

[26] Y. Yao and J. Gehrke, "The cougar approach to in-network query processing in sensor networks", in SIGMOD Record, September 2002.

[27] Qun Li, Javed Aslam, Daniela Rus, "Hierarchical Power aware Routing in Sensor Networks", In DIMACS Workshop on Pervasive Networking, Rutgers University, May 21, 2001. 